\title{
A Local Time Approach to the Self-Intersections of Brownian Paths in Space
}

Jay Rosen*

Department of Mathematics and Statistics, University of Massachusetts, Amherst, MA 01003, USA

Abstract. We study the Brownian functional

$$
\alpha(x, B)=\iint_{B} \int_{x}\left(W_{t}-W_{s}\right) d s d t,
$$

where $W_{t}$ is a Brownian path in two or three dimensions. For $B$ off the diagonal we identify $\alpha(x, B)$ with a local time, and establish the Hölder continuity of $\alpha(x, B)$ in both $x$ and $B$.

\section{Introduction}

A classical theorem of Dvoretzky, Erdös and Kakutani [1950] states that a Brownian path $W_{t}$ will intersect itself in both two and three dimensions. This fact is at the heart of Symanzik's approach to Euclidean quantum field theory [1969] where the key role is played by the purely formal expression

$$
\int_{0}^{h} \int_{0}^{h} \delta_{x}\left(W_{t}-W_{s}\right) d s d t
$$

Here $\delta_{x}$ is the Dirac delta function concentrated at $x$. When $x=0,(1.1)$ is meant as a measure of the amount of time $t, 0 \leqq t \leqq h$, spent by the path in intersecting itself. This expression also appears in the study of polymers, see Edwards [1965] and Westwater. In this paper we employ the general perspective of the theory of local times to analyze (1.1). For an excellent overview of local times, together with extensive references, we refer to the survey paper of Geman and Horowitz [1980].

The only general method for studying local times of Gaussian processes involves local non-determinism (LND), a concept introduced by Berman [1973], and generalized by Pitt [1978] and Cuzick. Since the process underlying (1.1) does not appear to be locally nondeterministic, we are forced to develop a new approach. We refer the reader to Rosen [1981], and Geman, Horowitz and Rosen [1981], where

* Partially supported by NSF-MCS-80-02940 
the local time of other processes are studied without using LND. In the latter paper we also discuss the question of the general role of LND.

Let us begin with the general definition of local time. If $X: R^{N} \rightarrow R^{d}$ is a Borel function, then for each Borel set $B \subset R^{N}$ we can define the occupation measure $\mu_{B}$ on $R^{d}$ by

$$
\mu_{B}(A)=\lambda_{N}\left(X^{-1}(A) \cap B\right) .
$$

Here $\lambda_{N}$ is Lebesgue measure on $R^{N}$. If $\mu_{B} \ll \lambda_{d}$ we say that $X$ has a local time on $B$, and define the local time on $B, \alpha(x, B)$, by

$$
\alpha(x, B) \doteq \frac{\mathrm{d} \mu_{B}}{d \lambda_{d}}(x) .
$$

Of course, this only defines $\alpha(x, B)$ a.e. $d \lambda_{d}(x)$. Intuitively, we think of $\alpha(x, B)$ as the amount of time in $B$ spent by our function at $x$. As a consequence of these definitions,

$$
\int_{R^{d}} f(x) \alpha(x, B) d x=\int_{B} f(X(T)) d \lambda_{N}(T)
$$

for all bounded Borel functions $f$. Finally, if $X$ is a random field, i.e. if $X=X(T, \omega)$, where $\omega$ is a point in a probability space $(\Omega, d \mathbb{P})$, we say that $X$ has a local time on $B$, if $X(\cdot, \omega)$ has a local time on $B$ for almost all $\omega$. We usually suppress $\omega$ in our formulas.

Now apply this setup to (1.1). Let $X: R_{+}^{2} \rightarrow R^{d}, d=2$ or 3 , be the random field defined by

$$
X(T)=W_{t}-W_{s}, \text { for } T=(s, t) .
$$

Here $R_{+}^{2}=\{(s, t) \mid s \geqq 0, t \geqq 0\}$. We have

Theorem 1. For any bounded Borel set $B \subseteq R_{+}^{2}, X$ has a local time on $B$, and $\alpha(x, B) \in L^{2}\left(R^{d}, d \lambda_{d}\right)$ a.s.

In particular, with $H \doteq[0, h]^{2}, \alpha(x, H) \in L^{2}\left(R^{d}, d \lambda_{d}\right)$. Formal expressions of the form (1.1), with $x=0$, are usually interpreted as

$$
\lim _{k \rightarrow \infty} \int_{0}^{h} \int_{0}^{h} g_{k}\left(W_{t}-W_{s}\right) d s d t=\lim _{k \rightarrow \infty} \int_{H} g_{k}(X(T)) d T,
$$

for a sequence of functions $g_{k}$ converging weakly to $\delta$. For simplicity we take $g_{k}(x)=\left(\frac{2 \pi}{k}\right)^{-d / 2} \exp \left(-k x^{2} / 2\right)$. Using (1.5) we have

$$
\lim _{k \rightarrow \infty} \int_{H} g_{k}(X(T)) d T=\lim _{k \rightarrow \infty} \int_{R^{d}} \alpha(x, H) g_{k}(x) d \lambda_{d}(x) .
$$

If $\alpha(x, H)$ were continuous at $x=0$, this limit would be simply $\alpha(0, H)$. Unfortunately, $\alpha(x, H)$ cannot be continuous at $x=0$, and in fact the limit in (1.7) is infinite. As we now explain, this is related to the fact that the image of a Brownian path in $R^{2}$ or $R^{3}$ has zero Lebesgue measure (Itô and McKean [1965]) and consequently $W_{t}$ itself 
does not have a local time. Let $v_{[0, h]}$ be the occupation measure for $W$ on $[0, h]$. Then

$$
\hat{\mu}_{H}(u)=\int_{0}^{h} \int_{0}^{h} e^{i u \cdot\left(W_{t}-W_{s}\right)} d s d t=\left|\hat{v}_{[0, h]}(u)\right|^{2},
$$

so that $\hat{\mu}_{H} \geqq 0$, and the monotone convergence theorem now shows that

$$
\begin{aligned}
& \lim _{k \rightarrow \infty} \int_{R^{d}} \alpha(x, H) g_{k}(x) d \lambda_{d}(x) \\
& =\lim _{k \rightarrow \infty}(2 \pi)^{-d} \int_{R^{d}} \alpha(x, H)\left(\int e^{i u \cdot x} e^{-u^{2} / 2 k} d u\right) d \lambda_{d}(x) \\
& =\lim _{k \rightarrow \infty}(2 \pi)^{-d} \int \hat{\mu}_{H}(u) e^{-u^{2} / 2 k} d u=(2 \pi)^{-d} \int \hat{\mu}^{H}(u) d u \\
& =\int\left|\hat{v}_{[0, h]}(u)\right|^{2} d u, \quad \text { by (1.8). }
\end{aligned}
$$

But, since $W_{t}$ does not have a local time, a fortiori $v_{[0, h]}$ does not have an $L^{2}$ density, so that (1.9) is infinite.

When $d=2$, Varadhan in an appendix to Symanzik [1969] has described a way to "renormalize" (1.1). Instead of using (1.6), which we saw gives $\infty$, Varadhan shows that it is possible to choose, independently of the path, a sequence of constants $c_{k} \rightarrow \infty$ such that $\int_{0}^{h} \int_{0}^{h} g_{k}\left(W_{t}-W_{s}\right) d t d s-c_{k} h$ converges in $L^{2}(d \mathbb{P})$. This "renormalized" version of (1.1) is acceptable for the purposes of quantum field theory.

In this paper we focus on a different, and complementary, aspect of (1.1). We shall see that the infinite result in (1.6), for both two and three dimensions, stems from the diagonal $\Delta$ in $H=[0, h]^{2}$. In fact, we will prove that for any bounded Borel set $B$ in $R_{+}^{2}(\varepsilon) \doteq\{(s, t)|s, t \geqq 0,| \mathrm{s}-\mathrm{t} \mid \geqq \varepsilon\}, \varepsilon>0$ arbitrary, $\alpha(x, B)$ is a continuous function of $x$, and then we find the simple identity $\int_{B} \int \delta_{x}\left(W_{t}-W_{s}\right) d s d t=\alpha(x, B)$. (By the continuity of the paths, these results persist for $x \neq 0$ even if $B \cap \Delta \neq \varnothing$.) In our opinion, given the work of Varadhan, the main obstruction to carrying out Symanzik's quantum field theory program is in understanding the behavior of $\alpha(x, B)$ for $B \subseteq R_{+}^{2}(\varepsilon)$. We present here a detailed analysis of the local behavior of $\alpha(x, B)$.

Our main theorem is

Theorem 2. If $B$ is a bounded Borel set in $R_{+}^{2}(\varepsilon)$ for some $\varepsilon>0$, then for any compact $K \subseteq R^{d}$ we can choose a version of $\alpha(x, B)$ such that a.s.

$$
\sup _{x, y \in K} \frac{|\alpha(x, B)-\alpha(y, B)|}{|x-y|^{\beta}}<\infty
$$

for any $\beta<1$ if $d=2$, and any $\beta<1 / 2$ if $d=3$.

Remark. Version refers to the fact, mentioned after (1.3), that $\alpha(x, B)$ can be altered on sets of measure zero $d \lambda_{d}(x)$. We can in addition require that for each fixed $x, \alpha\left(x,,^{\cdot}\right)$ be a finite measure on $R_{+}^{2}(\varepsilon)$.

In proving Theorem 2 we need the following result which is of interest by itself. 
Lemma 1. If $B$ is a bounded Borel set in $R_{+}^{2}(\varepsilon)$ for some $\varepsilon>0$, then for any $k$ we can find a version of $\alpha(x, B)$ with

$$
\alpha(x, B) \in L^{k}(d \mathbb{P}) \text { for all } x .
$$

A celebrated theorem of Trotter [1958] says that the local time $\alpha(x,[0, h])$ for (onedimensional) Brownian motion, $W_{t}$, is a continuous function of $(x, h)$. Our next theorem is an analogue of this. Let

$$
Q_{h_{1}, h_{2}}=\left\{(s, t)\left|0 \leqq s \leqq h_{1}, \quad 0 \leqq t \leqq h_{2},\right| s-t \mid \geqq \varepsilon\right\} .
$$

Theorem 3. We can choose a version of the local time such that, a.s., $\alpha\left(x, Q_{h_{1}, h_{2}}\right)$ is a continuous function of $\left(x, h_{1}, h_{2}\right)$.

We next study the behavior of $\alpha(x, B)$ as a function of $B \subseteq R_{+}^{2^{\prime}}(\varepsilon)$. Theorem 4 will follow easily from our results and the general theory.

Theorem 4. For each compact $K \subseteq R^{d}$, and any $\rho<1-d / 4$, there is a constant $c$, and a random variable $\delta=\delta(\omega)$ such that, a.s.,

$$
\alpha(x, B) \leqq c\left(\lambda_{N}(B)\right)^{\rho}, \quad \text { for all } x \in K,
$$

for any square $B \subseteq R_{+}^{2}(\varepsilon) \cap[0, h]^{2}$ of edge length less than $\delta$.

With more work we can show

Theorem 5. For each $x \in R^{d}, T \in R_{+}^{2}(\varepsilon)$ there exist a.s. finite random variables $c, \delta$ such that a.s.

$$
\alpha(x, B) \leqq c\left(\lambda_{N}(B)\right)^{1-d / 4}\left(\log \left|\log \lambda_{N}(B)\right|\right)^{d / 2}
$$

for any square $B \subseteq R_{+}^{2}(\varepsilon) \cap[0, h]^{2}$ of edge length less than $\delta$, with a corner at $T$.

Taylor [1966] for $d=2$, and Fristedt [1967] for $d=3$, have greatly improved on the above mentioned theorem of Dvoretzky, Erdös and Kakutani by showing that the (Hausdorff) dimension of the set of double points of a Brownian path is $4-\mathrm{d}$. (Wolpert [1978] has provided an alternate proof for $d=2$.) Again, our results together with the general theory will easily yield

Theorem 6. With probability one,

$$
\operatorname{dim}\left\{(s, t), s \neq t \mid W_{s}=W_{t}\right\}=2-d / 2 .
$$

Using Kaufman's ideas [1969] in the manner explained in Geman, Horowitz and Rosen [1981], we recover the Taylor-Fristedt result.

Theorem 7. With probability one,

$$
\operatorname{dim}\left\{x \mid x=W_{s}=W_{t}, s \neq t\right]=4-d .
$$

Finally, let $D=\left\{x \mid x=W_{s}=W_{t}, s \neq t\right\}$ be the set of double points. Using Theorem 5 and Kaufman [1969] we can show that, e.g. when $d=2, D$ has non-zero Hausdorff measure with respect to $t^{2}|\log t|^{6+\varepsilon}$. It is a conjecture of Taylor [1973] that $D$ has zero Hausdorff measure with respect to $t^{2}|\log t|^{2}$. We have no conjecture 
as to the correct Hausdorff measure function for $D$, but will put forward the conjecture that the correct measure function for $\left\{(s, t), s \neq t \mid W_{s}=W_{t}\right\}$ is that appearing in Theorem 5: $t^{2-d / 2}(\log |\log t|)^{d / 2}$.

\section{Proofs of Theorems 1-3, and Lemma 1}

Proof of Theorem 1. Let $B \subset[0, h]^{2}$ be a Borel set. It suffices to prove that

$$
\mathbb{E}\left(\int_{R^{d}}\left|\hat{\mu}_{B}(u)\right|^{2} d u\right)<\infty .
$$

From the definition (1.2) of $\mu_{B}$ we have

$$
\begin{aligned}
\mathbb{E}\left(\int_{R^{d}}\left|\hat{\mu}_{B}(u)\right|^{2} d u\right) & =\int_{R^{d}} \mathbb{E}\left(\left|\hat{\mu}_{B}(u)\right|^{2}\right) d u \\
& =\int_{R^{d}} \int_{B} \int_{B} \mathbb{E}\left(e^{i u \cdot\left(X(T)-X\left(T^{\prime}\right)\right)}\right) d T d T^{\prime} d u \\
& =\int_{R^{d}} \int_{B} \int_{B} e^{-(1 / 2) \vee\left(u \cdot\left(X(T)-X\left(T^{\prime}\right)\right)\right)} d T d T^{\prime} d u,
\end{aligned}
$$

where as usual $\mathbb{V}(x)=\mathbb{E}\left(x^{2}\right)-\mathbb{E}^{2}(x)$. Let $T=\left(t_{1}, t_{2}\right), T^{\prime}=\left(t_{3}, t_{4}\right)$. If $\pi$ denotes a permutation of $(1,2,3,4)$, let $\Delta(\pi)$ be the set of $\left(t_{1}, t_{2}, t_{3}, t_{4}\right) \in R^{4}$ such that

$$
t_{\pi(1)}<t_{\pi(2)}<t_{\pi(3)}<t_{\pi(4)} \text {. }
$$

By checking the few possibilities it is easy to verify that

$$
\mathbb{V}\left(u \cdot\left(X(T)-X\left(T^{\prime}\right)\right)\right)=|u|^{2}\left[\left(t_{\pi(2)}-t_{\pi(1)}\right)+\left(t_{\pi(4)}-t_{\pi(3)}\right)\right]
$$

for $\left(T, T^{\prime}\right) \in \Delta(\pi)$. Changing variables

$$
\left(t_{1}, t_{2}, t_{3}, t_{4}\right) \rightarrow\left(t_{\pi(1)}, t_{\pi(2)}-t_{\pi(1)}, t_{\pi(3)}-t_{\pi(2)}, t_{\pi(4)}-t_{\pi(3)}\right) .
$$

we see that the integral in (2.2) over $(B \times B) \cap \Delta(\pi)$ is bounded by

$$
\int_{R^{d}} \int_{0}^{h} \cdots \int_{0}^{h} e^{-(1 / 2)|u|^{2}\left(s_{2}+s_{4}\right)} d s_{1} \cdots d s_{4} d u<c \int_{R^{d}}\left(1+|u|^{2}\right)^{-2} d u,
$$

which is finite for $d<4$. In (2.4) we used the bound

$$
\int_{0}^{h} e^{-|u|^{2} s} d s=\frac{1-e^{-h|u|^{2}}}{|u|^{2}} \leqq c\left(1+|u|^{2}\right)^{-1} .
$$

Thus (2.2) is finite over each $(B \times B) \cap \Delta(\pi)$, and there are only a finite number of permutations.

Proof of Lemma 1. This follows from the arguments used in proving Lemma 1 of Rosen [1981] and the following lemma which is basic for all our results.

Lemma 2. Let $B$ be a bounded Borel set in $R_{+}^{2}(\varepsilon)$ for some $\varepsilon>0$. Then

$$
\int_{R^{d k}} \int_{B^{k}} \prod_{l=1}^{k}\left|u^{l}\right|^{\gamma} \exp \left[-(1 / 2) \mathbb{V}\left(\sum_{j=1}^{k} u^{j} \cdot X\left(T^{j}\right)\right)\right] d T d u<\infty
$$


for all $k \in \mathbb{Z}_{+}$and any $\gamma<\left\{\begin{array}{ll}1 & \text { if } d=2 \\ 1 / 2 & \text { if } d=3\end{array}\right.$.

In Lemma $2, u^{1}, \ldots u^{k} \in R^{d}, T^{1}, \ldots, T^{k} \in R^{2}$, and $d T=\prod_{j=1}^{k} d T^{j}, \quad d u=\prod_{j=1}^{k} d u^{j}$. Section 3 is devoted to proving this lemma.

Proof of Theorems 2 and 3. Using Lemmas 1 and 2 we can show that for any $x$ and $y$

$$
\begin{aligned}
\mathbb{E}\left([\alpha(x, B)-\alpha(y, B)]^{k}\right)= & (2 \pi)^{-d} \int_{R^{d k}} \prod_{j=1}^{k}\left(e^{-i u^{j} \cdot X}-e^{-i u^{j} \cdot Y}\right) \\
& \times \int_{B^{k}} \exp \left[-(1 / 2) \mathbb{V}\left(\sum_{j=1}^{k} u^{j} \cdot X\left(T^{j}\right)\right)\right] d T d u .
\end{aligned}
$$

The details are spelled out in the proof of Lemma 1 of Rosen [1981]. Using the bound $\left|e^{-i u^{j} \cdot X}-e^{-i u^{j} \cdot Y}\right|<\left|u^{j}\right|^{\gamma}|x-y|^{\gamma}$ for any $\gamma<1$, together with Lemma 2 shows that for any $k \in \mathbb{Z}_{+}$

$$
\mathbb{E}\left([\alpha(x, B)-\alpha(y, B)]^{k}\right) \leqq c|x-y|^{k \gamma} \text { for } \gamma<\left\{\begin{array}{ll}
1 & \text { if } d=2 \\
1 / 2 & \text { if } d=3
\end{array} .\right.
$$

This is an "integrated" version of Theorem 2 . To go from this to Theorems 2 and 3 we need only refer to Sect. 27 of Geman and Horowitz [1980].

\section{Proof of Lemma 2.}

Proof of Lemma 2. Let $T^{j}=\left(s_{j}, t_{j}\right)$ and write $\left(z_{1}, z_{2}, \ldots, z_{2 k}\right)=\left(s_{1}, t_{1}, s_{2}, t_{2}, \ldots s_{k}, t_{k}\right)$. We may assume $B \subset\{(s, t) \mid t>s\}$, for otherwise, in the region $s_{j}>t_{j}$, change $u^{j}$ to $-u^{j}$. We may also assume that $B^{k}$ is replaced by $B^{k} \cap \Delta(\pi)$ for some permutation $\pi$ of $\{1, \ldots, 2 k\}$, where $\Delta(\pi)=\left\{\left(z_{1}, \ldots, z_{k}\right) \mid z_{\pi(1)}<z_{\pi(2)}<\ldots z_{\pi(2 k)}\right\}$, since $(2.6)$ is a sum of integrals over such regions.

Let us define disjoint intervals

$$
R_{i}=\left[z_{\pi(i)}, z_{\pi(i+1)}\right], \quad 1 \leqq i \leqq 2 k-1 .
$$

Setting $X\left(R_{i}\right)=X\left(z_{\pi(i)}, z_{\pi(i+1)}\right)$, we see that

$$
X\left(T^{J}\right)=\sum_{R_{\imath} \subseteq\left[s_{j}, t_{j}\right]} X\left(R_{i}\right)
$$

The $X\left(R_{i}\right)$ are independent so that

$$
\mathbb{V}\left(\sum_{j=1}^{k} u^{j} \cdot X\left(T^{j}\right)\right)=\mathbb{V}\left(\sum_{j=1}^{k} u^{j} \cdot \sum_{R_{\imath} \subseteq\left[s_{j}, t_{j}\right]} X\left(R_{i}\right)\right)=\sum_{i}\left|\bar{u}^{i}\right|^{2}\left|R_{i}\right|
$$

on introducing the notation

$$
\bar{u}^{i}=\sum_{j: R_{\imath} \subseteq\left[s_{j}, t_{j}\right]} u^{j}
$$


For later use, we let $R_{0}$ be an interval on the left of $R_{1}$, and $R_{2 k}$ an interval on the right of $R_{2 k-1}$. We set $\bar{u}^{0}=0, \bar{u}^{2 k}=0$.

By (3.3), to prove (2.6) it suffices to show that

$$
\int_{R^{d k}} \int_{B^{k} \cap \Delta(\pi)} \prod_{l=1}^{k}\left|u^{l}\right|^{\gamma} \exp \left(-\sum_{i}\left|\bar{u}^{i}\right|^{2}\left|R_{i}\right|\right) d T d u<\infty .
$$

From this, it is clear that we may assume $\bigcup_{J}\left[s_{j}, t_{j}\right]$ connected - by working with each component separately.

Each interval $\left[s_{l}, t_{l}\right]$ is a union of adjacent $R_{i}$. We define $f(l)$ and $r(l)$ by requiring that $R_{f(l)}$ is the first and $R_{r(l)-1}$ the last of the $R_{i}$ intervals in $\left[s_{l}, t_{l}\right]$. Thus

$$
\left\{s_{l}, t_{l}\right\}=R_{f(l)} \cup R_{f(l)+1} \cup \ldots \cup R_{r(l)-1} .
$$

Equivalently, $z_{\pi f(l)}=s_{l}, z_{\pi r(l)}=t_{l}$.

Clearly from (3.4)

$$
\left\{\begin{aligned}
u^{l} & =\bar{u}^{f(l)}-\bar{u}^{f(l)-1} \\
-u^{l} & =\bar{u}^{r(l)}-\bar{u}^{r(l)-1},
\end{aligned}\right.
$$

and therefore

$$
\left|u^{l}\right| \leqq\left(\left|\bar{u}^{f(l)}\right|+\left|\bar{u}^{f(l)-1}\right|\right)^{1 / 2}\left(\left|\bar{u}^{r(l)}\right|+\left|\bar{u}^{r(l)-1}\right|\right)^{1 / 2} .
$$

Since the $2 k$ points $s_{i}, t_{j}$ can be assumed distinct, the maps

$$
\begin{gathered}
f:\{1, \ldots, k\}+\{1, \ldots, 2 k-2\}, \\
r:\{1, \ldots, k\}+\{2, \ldots, 2 k],
\end{gathered}
$$

are injective with disjoint range. Now (3.8) yields

$$
\begin{aligned}
\prod_{l=1}^{k}\left|u^{l}\right| & \leqq \prod_{l=1}^{k}\left(\left|\bar{u}^{f(l)}\right|+\left|\bar{u}^{f(l)-1}\right|\right)^{1 / 2}\left(\left|\bar{u}^{r(l)}\right|+\left|\bar{u}^{r(l)-1}\right|\right)^{1 / 2} \\
& \leqq c \prod_{i=1}^{2 k-1}\left(1+\left|\bar{u}^{i}\right|^{2}\right)^{1 / 2}
\end{aligned}
$$

since each $i$ is either an $f(l)$ or an $r(l)$ for a unique $l$, and either an $f(m)-1$ or an $r(m)-1$ for a unique $m$, and we always have $x<1+x^{2}$.

To estimate the inner integral in (3.5),

$$
\int_{B^{k} \cap \Delta(\pi)} \exp \left(-\sum_{i}\left|\bar{u}^{i}\right|^{2}\left|R_{i}\right|\right) d T,
$$

we first change variable: $\left(T^{1}, \ldots, T^{k}\right) \rightarrow\left(z_{\pi(1)},\left|R_{1}\right|, R_{2}|, \ldots,| R_{2 k-1}\left|R_{2 k-1}\right|\right)$. Since $\left|s_{j}-t_{j}\right| \geqq \varepsilon$, for each $j, 1 \leqq j \leqq k$, we can choose some $R_{d(j)} \subseteq\left[s_{j}, t_{j}\right]$ such that $\left|R_{d(j)}\right| \geqq \varepsilon / 2 k$. (Note: the map $d(\cdot)$ is not necessarily injective.) For the $d\left|R_{d(j)}\right|$ integral we use

$$
\int_{\varepsilon / 2 k}^{\infty} e^{-\mid u^{\left.d(j)\right|^{2} t}} d t \leqq c e^{-\bar{c} \mid \bar{u}^{d(j) \mid}} \leqq c\left(1+\left|\bar{u}^{d(j)}\right|^{2}\right)^{-2 k}
$$


For the other integrals we use (if $B \subseteq[0, h]^{2}$ )

$$
\int_{0}^{h} e^{-\left|u^{1}\right| 2 t} d t=\frac{1-e^{-h\left|\bar{u}^{1}\right| 2}}{\left|\bar{u}^{i}\right|^{2}} \leqq c\left(1+\left|\bar{u}^{i}\right|^{2}\right)^{-1} .
$$

Using (3.9), (3.11), (3.12) we see that the integral in (3.5) is bounded by

$$
\begin{aligned}
\int_{R^{d k}} & \prod_{j=1}^{k}\left(1+\left|\bar{u}^{f(j)}\right|^{2}\right)^{-1+\gamma / 2}\left(1+\left|\bar{u}^{r(j)}\right|^{2}\right)^{-1+\gamma / 2}\left(1+\left|\bar{u}^{d(j)}\right|^{2}\right)^{-1} d u \\
\leqq & {\left[\int_{R^{d k}} \prod_{j=1}^{k}\left(1+\left|\bar{u}^{f(j)}\right|^{2}\right)^{-2+\gamma} d u\right]^{1 / 2} } \\
& \times\left[\int_{R^{d k}} \prod_{j=1}^{k}\left(1+\left|\bar{u}^{r(j)}\right|^{2}\right)^{-2+\gamma}\left(1+\left|\bar{u}^{d(j)}\right|^{2}\right)^{-2} d u\right]^{1 / 2} .
\end{aligned}
$$

We will prove the following simple lemma.

Lemma 3. The $\bar{u}^{f(1)}, \bar{u}^{f(2)}, \ldots, \bar{u}^{f(k)}$ are a nonsingular set of coordinates for $R^{d k}$.

By this lemma, changing coordinates in the first integral of the bottom line of (3.13), we see that this integral is finite if

$$
\int_{R^{d}}\left(1+|y|^{2}\right)^{-2+\gamma} d y
$$

which means $d<4-2 \gamma$, i.e. $\gamma<2-d / 2$.

The second integral is handled similarly with the help of the following less simple lemma. This will complete the proof of Lemma 2.

Lemma 4. We can choose a nonsingular set of coordinates for $R^{d k}$ from the set

$$
\bar{u}^{r(1)}, \bar{u}^{r(2)}, \ldots, \bar{u}^{r(k)}, \bar{u}^{d(1)}, \bar{u}^{d(2)}, \ldots, \bar{u}^{d(k)} \text {. }
$$

Before proving Lemmas 3 and 4 we offer a simple illustration. Let $k=2$ and $s_{1}<s_{2}<t_{2}<t_{1}$. Then $\bar{u}^{1}=u^{1}, \bar{u}^{2}=u^{1}+u^{2}, \bar{u}^{3}=u^{1}, \bar{u}^{4}=0, f(1)=1, f(2)=2$ and clearly $\left\{\bar{u}^{1}, \bar{u}^{2}\right\}$ are a nonsingular set of coordinates for $R^{2 d}$. Here $r(1)=4$, $r(2)=3, d(1)=d(2)=2$, and we see again that $\left\{\bar{u}^{3}, \bar{u}^{2}\right\}$ are a nonsingular set of coordinates for $R^{2 d}$.

Proof of Lemma 3. Let $p$ be the permutation of $\{1, \ldots, k\}$ such that $f p(1)<f p(2)<\ldots$ $<f p(k)$. We prove our lemma by showing inductively that

$$
\operatorname{span}\left\{\bar{u}^{f p(j)} \mid j \leqq l\right\}=\operatorname{span}\left\{u^{p(j)} \mid j \leqq l\right\} \text { for } l=1, \ldots, k
$$

Equation (3.14) is true for $l=1$, since necessarily $\bar{u}^{f p(1)}=\bar{u}^{1}=u^{p(1)}$. Assume (3.14) for $l=1, \ldots, m-1$. Then by the definitions of $f$ and $p$

$$
\bar{u}^{f p(m)}-u^{p(m)} \in \operatorname{span}\left\{u^{p(j)} \mid j \leqq m-1\right\},
$$

so that (3.14) holds for $l=m$ if it holds for $l=m-1$. 
Proof of Lemma 4. We cannot expect $\bar{u}^{r(1)}, \bar{u}^{r(2)}, \ldots, \bar{u}^{r(k)}$ alone to be a non-singular set of coordinates since $\bar{u}^{r(k)}=\bar{u}^{2 k}=0$. We begin by showing

$$
\operatorname{span}\left\{\bar{u}^{r(1)}, \bar{u}^{r(1)}, \bar{u}^{r(2)}, \ldots, \bar{u}^{r(k)}\right\}=\operatorname{span}\left\{u^{i} \mid\left[s_{i}, t_{i}\right] \cap\left(\bigcup_{j} R_{r(j)}\right) \neq \varnothing\right\}
$$

A more suggestive way of expressing (3.15) is to call $u^{j}$ a component of $\bar{u}^{i}$ if it appears in (3.4), and then (3.15) says that the span of $\left\{\bar{u}^{r(1)}, \ldots, \bar{u}^{r(k)}\right\}$ is equal to the span of its components.

Let $q$ be the permutation of $\{1, \ldots, k\}$ such that $r q(1)<r q(2)<\ldots<r q(k)$. We establish (3.15), by showing, using downward induction, that

$$
\operatorname{span}\left\{\bar{u}^{r q(j)} \mid j \geqq l\right\}=\operatorname{span}\left\{u^{i} \mid\left[s_{i}, t_{i}\right] \cap\left(\bigcup_{j \geq l} R_{r q(j)}\right) \neq \varnothing\right\}, \quad l=1, \ldots, k .
$$

Equation (3.16) is easy for $l=k$, since $r q(k)=2 k$, and both spans are 0 .

Assume (3.16) for $l=m+1$. Then by the definitions of $r$ and $q$ the components of $\bar{u}^{r q(m)}$ with the one possible exception of $u^{q(m+1)}$ are included among the components of $\bar{u}^{r q(m+1)}$, so that (3.16) is true for $l=m$ if it is true for $l=m+1$. This proves (3.15).

We now study these $u^{i}$ which do not appear in (3.15). Consider an $i$ such that $\left[s_{i}, t_{i}\right] \cap \bigcup_{j} R_{r(j)}=\varnothing$. Since $R_{d(i)} \subseteq\left[s_{i}, t_{i}\right]$, we have that $u^{i}$ is a component of $\bar{u}^{d(i)}$. Since $u^{i}$ is not a component of any $\bar{u}^{r(\cdot)}$, we must have (if $r(l)$ is the first of the $r$ 's with $d(i)<r(l))$ that the component of $\bar{u}^{d(l)}-u^{i}$ are included among the components of $\bar{u}^{r(l)}$. Hence from $(3.15)$

$$
\operatorname{span}\left\{\bar{u}^{r(1)}, \ldots, \bar{u}^{r(k)}, \bar{u}^{d(i)}\right\} \supseteq\left\{u^{i}\right\}
$$

This now shows

$$
\operatorname{span}\left\{\bar{u}^{r(1)}, \ldots, \bar{u}^{r(k)}, \bar{u}^{d(1)}, \ldots, \bar{u}^{d(k)}\right\}=R^{d k}
$$

and proves Lemma 4.

\section{Proofs of Theorems 4,5 and 6}

Proof of Theorem 4. Consider the inner integral in (3.5)

$$
\int_{B^{k} \cap \Delta(\pi)} \exp \left(-\sum_{i}\left|\bar{u}^{i}\right|^{2}\left|R_{i}\right|\right) d T .
$$

Let $p$ be any real number such that $1 / p<1-d / 4$. Then if $1 / p+1 / q=1$, we have

$$
d<4 / q
$$

Applying Hölder's inequality to (4.1) we find

$$
\int_{B^{k} \cap \Delta(\pi)} \exp \left(-\sum_{i}\left|\bar{u}^{i}\right|^{2}\left|R_{i}\right|\right) d T \leqq\left(\lambda_{N}(B)\right)^{k / p}\left(\int_{B^{k} \cap \Delta(\pi)} \exp \left(-\sum_{i}\left|\bar{u}^{i}\right|^{2}\left|R_{i}\right|\right) d T\right)^{1 / q} \text {. }
$$


The proof of Lemma 2 shows that

$$
\int_{R^{d k}} \prod_{l=1}^{k}\left|u^{l}\right|^{\gamma}\left(\int_{B^{k} \cap \Delta(\pi)} \exp \left(-q \sum_{i}\left|\bar{u}^{i}\right|^{2}\left|R^{i}\right|\right) d T\right)^{1 / q}<\infty
$$

as long as

$$
d<4 / q-2 \gamma .
$$

By (4.2) we can choose $\gamma$ so small that (4.4) holds. As in Lemma 1 and Theorem 2 this implies

$$
\mathbb{E}\left([a(x, B)]^{k}\right) \leqq c\left(\lambda_{N}(B)\right)^{k / p},
$$

and

$$
\mathbb{E}\left([a(x, B)-a(y, B)]^{k}\right) \leqq c\left(\lambda_{N}(B)\right)^{k / p}|x-y|^{k \gamma} .
$$

With these two inequalities, the proof of our theorem now follows from Sect. 27 of Geman and Horowitz [1980].

Proof of Theorem 5. Let $B=[a, a+h] \times[b, b+h]$. We can assume $a<b$, and $h$ so small that $a+h+\varepsilon / 2 \leqq b$. In this situation we can make a more detailed analysis of

$$
\begin{aligned}
\mathbb{E}\left([a(x, B)]^{k}\right) & \leqq(2 \pi)^{-d k} \int_{B^{k}} \int_{R^{d k}} \exp \left[-(1 / 2) V\left(\sum_{j=1}^{k} u^{j} \cdot X\left(T^{j}\right)\right)\right] d u d T \\
& =(2 \pi)^{-d k} \sum_{\pi} \int_{B^{k}} \int_{\Delta(\pi) R^{d k}} \exp \left[-(1 / 2) \sum\left|\bar{u}^{i}\right|^{2}\left|R_{i}\right|\right] d u d T,
\end{aligned}
$$

where we are using the notation of the proofs of Theorem 2 and Lemma 2. Note that in our case (B a square) all $s_{i}<t_{j}$. Let $\pi_{1}, \pi_{2}$ be permutations of $\{1, \ldots, k]$, and define

$$
\begin{aligned}
& \Delta\left(\pi_{1}\right)=\left\{\left(s_{1}, \ldots, s_{k}\right) \mid s_{\pi_{1}(1)}<s_{\pi_{1}(2)}<\ldots<s_{\pi_{1}(k)}\right\}, \\
& \Delta\left(\pi_{2}\right)=\left\{\left(t_{1}, \ldots, t_{k}\right) \mid t_{\pi_{2(1)}}<t_{\pi_{2}(2)}<\ldots<t_{\pi_{2}(k)}\right\},
\end{aligned}
$$

respectively. Then in $\Delta\left(\pi_{1}\right) \times \Delta\left(\pi_{2}\right)$ we have

$$
\bar{u}^{l}=\left\{\begin{array}{l}
\sum_{j=1}^{l} u^{\pi_{1}(j)}, \quad 1 \leqq i \leqq k \\
\sum_{J=l}^{k} u^{\pi_{2}(j)}, \quad k+1 \leqq i \leqq 2 k-1
\end{array}\right.
$$

so that

$$
\begin{aligned}
& E\left([a(x, B)]^{k}\right) \leqq \\
& \quad \leqq(2 \pi)^{-d k} \sum_{\left.\pi_{1}, \pi_{2} B^{k} \cap\left(\Delta\left(\pi_{1}\right) \times \Delta\left(\pi_{1}\right)\right)\right)} \int_{R^{d k}} \exp \left[-\sum_{i=1}^{2 k-1}\left|\bar{u}^{i}\right|^{2}\left|R_{i}\right| / 2\right] d u d T \\
& \quad \leqq(2 \pi)^{-d k} \sum_{\pi_{1}, \pi_{2} B^{k} \cap\left(\Delta\left(\pi_{1}\right) \times \Delta\left(\pi_{2}\right)\right)}\left(\int_{R^{d k}} \exp \left[-\sum_{i=1}^{k}\left|\bar{u}^{i}\right|^{2}\left|R_{i}\right| / 2\right]\right)^{1 / 2}
\end{aligned}
$$




$$
\begin{aligned}
& \times\left(\int_{R^{d k}} \exp \left[-\sum_{i=k}^{2 k-1}\left|\bar{u}^{i}\right|^{2}\left|R_{i}\right| / 2\right]\right)^{1 / 2} d T \\
\leqq & (2 \pi)^{-d k / 2} \sum_{\pi_{1}, \pi_{2} B^{k} \cap\left(\Delta\left(\pi_{1}\right) \times \Delta\left(\pi_{2}\right)\right.} \prod_{i=1}^{k}\left|R_{i}\right|^{-d / 4} \prod_{j=k}^{2 k-1}\left|R_{j}\right|^{-d / 4} d T .
\end{aligned}
$$

But since $t_{\pi_{2}(1)}-S_{\pi_{1}(k)} \geqq \varepsilon / 2$, the last line is then

$$
\begin{aligned}
\leqq & c^{k} \sum_{\pi_{1}, \pi_{2}}\left(\int_{[a, a+h]^{k} \cap \Delta\left(\pi_{1}\right)} \sum_{i=1}^{k-1}\left|R_{i}\right|^{-d / 4} d s_{1} \ldots d s_{k}\right) \\
& \times\left(\int_{[b, b+h]^{k} \cap \Delta\left(\pi_{2}\right)} \sum_{j=k+1}^{2 k-1}\left|R_{j}\right|^{-d / 4} d t_{1} \ldots d t_{k}\right) \\
\leqq & c^{k}(k !)^{2}\left(h \int_{0<t_{1}<t_{2}<\ldots<t_{k-1} \leqq h} \prod_{i=1}^{k-1} \frac{d t_{i}}{\left(t_{i}-t_{i-1}\right)^{d / 4}}\right)^{2},
\end{aligned}
$$

and by the lemma in Kono [1977] this is

$$
\begin{aligned}
& \leqq c^{k}(k !)^{2}\left(\frac{h \cdot h^{(k-1)(1-d / 4)}((k-1) !)^{d / 4}}{(k-1) !}\right)^{2} \\
& \leqq c^{k} h^{k(2-d / 2)}(k !)^{d / 2}
\end{aligned}
$$

Our theorem now follows as in Kono [1977]. (Although this work seems to require $d / 2<1$, with our estimate it can easily be modified.)

Proof of Theorem 6. Let us first use an argument of Tran [1976], to show that, a.s.,

$$
\alpha\left(0, R_{+}^{2}(\varepsilon)\right)>0 \text {. }
$$

Let $S_{n}=\left\{(s, t)|n-1 \leqq s, t \leqq n| s-t \mid, \geqq \frac{1}{2}\right\}$. Note that $\alpha\left(0, S_{n}\right)$ are independent identically distributed random variables, since Brownian motion has independent increments. Furthermore

$$
E\left(\alpha\left(0, S_{1}\right)\right)=(2 \pi)^{-1} \iint_{R^{d}} \int_{S_{1}} e^{-(1 / 2)|u|^{2}|t-s|} d s d t d u>0
$$

Hence if $A_{n}=\left\{\alpha\left(0, S_{n}\right)>0\right\}$, we have

$$
P\left(A_{n}\right)=\delta>0
$$

for some $\delta>0$, and all $n=1,2, \ldots$ The Borel-Cantelli lemma then shows

$$
P\left(A_{n} \text { infinitely often }\right)=1 \text {. }
$$

which implies (4.5).

We now follow Adler [1978], easily modified to cover $d \neq 1$. His Lemma 5 together with (4.5) and our Theorem 4 show that, a.s., for every $\varepsilon>0$

$$
\operatorname{dim}\left\{(s, t) \in R_{+}^{2}(\varepsilon) \mid W_{s}=W_{t}\right\} \geqq 2-d / 2 .
$$


On the other hand, the Brownian path is Hölder continuous of any order $<\frac{1}{2}$, so that Adler's Lemma 7, together with our Theorem 2, shows that, a.s., for any $\varepsilon>0$

$$
\operatorname{dim}\left\{(s, t) \in R_{+}^{2}(\varepsilon) \mid W_{s}=W_{t}\right\} \leqq 2-d / 2 .
$$

These prove Theorem 6 .

Acknowledgement. It is a pleasure to thank D. Geman and J. Horowitz for many helpful conversations.

\section{References}

Adler, R. (1978): The uniform dimension of the level sets of a Brownian sheet. Ann. Prob. 6, 509-515 Berman, S. (1973): Local nondeterminism and local times of Gaussian processes. Ind. Univ. Math. J. 23, 69-94

Cuzick, J.: Continuity of Gaussian local times, Ann. Prob. (to appear)

Dvoretzky, A., Erdös, P., Katutani, S. (1950): Double points of paths of Brownian motion in n-space. Acta Sci. Math. Szeged, 12B, 75-81

Edwards, S. (1965): The statistical mechanics of polymers with excluded volume. Proc. Phys. Sci. 85, 613-624

Fristedt, B. (1967): An extension of a theorem of S. J. Taylor concerning the multiple points of the symmetric processes. Z. Wahrsch. Verw. Gebiete 9, 62-64

Geman, D., Horowitz, J. (1980): Occupation densitıes, Ann. Probability 8, 1-67

German, D., Horowitz, J., Rosen, J.: The local time of intersections for Brownian paths in the plane. Ann. Prob. (to appear).

Itô, K., McKean, H. P. (1965): Diffusion processes and their sample paths. New York: Academic Press Kaufman, R. (1969): Une propriété métrique des mouvement brownien. C. R. Acad. Sc. Parıs, t. 268, Sérıe A, $727-728$

Kono, N. (1977): Hölder conditions for the local times of certain Gaussian processes with stationary increments. Proc. Jpn. Acad. 53, Ser. A, No. 3, 84-87

Pitt, L. (1978): Local times for Gaussian vector fields. Ind. Univ. Math. J. 27, 309-330

Rosen, J.(1981): Joint continuity of the local time for the N-parameter Wiener process in $R^{d}$. University of Massachusetts preprint

Symanzik, K. (1969): Euclidean quantum field theory. In: Local Quantum Theory, Jost, R. (ed.) New York: Academıc Press

Taylor, S. J. (1966): Multiple points for the sample paths of the symmetric stable processes. Z. Wahrsch. Verw. Gebiete 5, 247-264

Taylor, S. J. (1973): Sample path properties of processes with stationary independent increments. In: Stochastic Analysis, Kendall, D., Harding, E. (eds.) London: J. Wiley and Sons

Tran, L. T. (1976): On a problem posed by Orey and Pruitt related to the range of the N-parameter Wiener process in $\mathbb{R}^{d}$. Z. Wahrsch. Verw. Gebiete 6, 170-180

Trotter, H. (1958): A property of Brownian motion paths. Ill. J. Math. 2, 425-432

Westwater, J.: On Edwards' model for long polymer chains. Univ. of Washington preprint

Wolpert, R. (1978): Wiener path intersections and local time. J. Funct. Anal. 30, 329-340

Communicated by A. Jaffe

Received March 29, 1982; in revised form October 4, 1982 